\title{
Simulação computacional aplicada na análise do projeto de um restaurante universitário
}

\author{
José Victor Silvério ${ }^{1}$ \\ Stella Jacyszyn Bachega ${ }^{1}$ \\ Dalton Matsuo Tavares ${ }^{2}$ \\ Sílvia Parreira Tannús ${ }^{1}$ \\ Ricardo Ribeiro Moura ${ }^{1}$ \\ Sérgio Antônio Ribeiro de Carvalho Júnior ${ }^{3}$
}

\begin{abstract}
Resumo: o uso da simulação computacional foi impulsionado pelos avanços da computação e sua grande flexibilidade de utilização. Dentre as diversas aplicações, a análise de projetos ganha destaque. O presente trabalho tem como objetivo aplicar a simulação computacional para análise da capacidade de atendimento de um novo restaurante universitário. Para tanto, foram utilizados a abordagem de pesquisa quantitativa e o procedimento de pesquisa experimental, por meio do uso de simulação computacional. Mediante a análise dos resultados do modelo computacional desenvolvido, foi possível compreender o provável funcionamento do sistema estudado, bem como o fluxo das entidades entre os locais de atendimento. Verificou-se que o espaço físico projetado é adequado para o atendimento. No entanto, o processo de atendimento do local caixa deverá ser revisto, pois em horários de maior procura pelo serviço houve grande formação de fila de espera. Este trabalho apresenta contribuições para a área acadêmica e para a área empresarial.
\end{abstract}

Palavras-chave: Simulação computacional. Análise de projeto. Restaurante universitário.

\begin{abstract}
: the use of computer simulation was driven by advances in computer science and its great flexibility of use. Among the various applications, the project analysis is highlighted. This paper aims to apply the computer simulation to analyze the service capacity of a new university restaurant. Therefore, we used the quantitative survey approach and the experimental research procedure by means of computer simulation. By analyzing the results of the developed computational model, it was possible to understand the likely operation of the studied system and the flow of entities between service counters. It was verified that the projected physical space is suitable for the service. However, the attendance process for the local counter should be revised, because in times of increased demand for the service, there was a great waiting queue formation. This paper presents contributions to the academic and business areas.
\end{abstract}

Keywords: Computer simulation. Design analysis. University restaurant.

\section{Introdução}

A aplicação da simulação computacional para análise de cenários e auxílio no processo decisório tem sido crescente [1] [2]. Tal crescimento pode ser atribuído em parte ao largo desenvolvimento da computação [3], bem

\footnotetext{
${ }^{1}$ Faculdade de Engenharia (FENG), Curso de Engenharia de Produção, Universidade Federal de Goiás - Regional Catalão (UFG-RC), Catalão (GO) - Brasil

\{josevictorsilverio@gmail.com, stella@ufg.br, silviaparreira@yahoo.com.br, ricardoribeiromoura@gmail.com\}

${ }^{2}$ Instituto de Biotecnologia (IBioTec), Curso de Ciência da Computação, Universidade Federal de Goiás - Regional Catalão (UFG-RC), Catalão (GO) - Brasil

\{dalton_tavares@ufg.br\}

${ }^{3}$ Departamento de Compras, Universidade Federal de Goiás - Regional Catalão (UFG-RC), Catalão (GO) - Brasil

\{sergio_junior@ufg.br\}
}

http://dx.doi.org/10.5335/rbca.v8i2.5553

Revista Brasileira de Computação Aplicada (ISSN 2176-6649), Passo Fundo, v. 8, n. 2, p. 99-112, jul. 2016 
como sua flexibilidade de utilização, que varia desde operações na área de saúde, tecnologia a operações militares [4].

A simulação computacional é uma técnica poderosa aplicada à análise de processos e sistemas com altos níveis de complexidade [5] e incerteza [6]. Consiste em uma das técnicas de pesquisa operacional e envolve conceitos como o método de Monte Carlo e a Teoria das Filas. Torga [7] ressalta que tanto sistemas reais quanto conceituais fazem parte do escopo dessa técnica.

O estudo dos sistemas conceituais foi destacado por Jahangirian et al. [8] como uma das áreas da simulação com quantidade expressiva de publicações. Tais publicações retratam aplicações em planejamento e controle de projetos/sistemas conceituais [9] [10], sistemas multiagentes [11], protocolos de comunicação [12], tráfego multimídia em redes IP [13], balanceamento e projeto de linhas de produção [14] [15], etc. Di Gironimo et al. [1] destacam que a simulação representa uma ajuda valiosa na análise de cenários e na mensuração ou validação de soluções.

Dentre as aplicações da simulação computacional em sistemas conceituais pode-se destacar o estudo de Swart e Donno [9]. Houve a aplicação da simulação para análise de novas unidades da rede de fast food Burger King. Foram analisados o potencial de vendas, capacidade da cozinha, velocidade dos serviços, entre outros aspectos, visando o dimensionamento adequado de novas lojas.

Já Sánchez, Ríos e Malo [10] usam a simulação computacional para o redesenho de um restaurante. Neste estudo, motivados pela redução das vendas devido ao longo tempo de espera dos clientes, os autores buscaram um novo arranjo físico para o restaurante, que fosse capaz de otimizar a utilização de mão de obra e reduzir a movimentação dos colaboradores. Jansen et al. [6] e Van der Vorst, Beulens e Van Beek [16] empregaram a simulação computacional no projeto e redesenho de novas redes ou alternativas de distribuição de alimentos, buscando a redução do tempo de entrega e aumentando a eficiência dos processos logísticos.

Como observado, as aplicações da simulação computacional são diversas e buscam analisar alternativas ao modelo padrão já utilizado ou a criação de um novo sistema. Com base nesse contexto e justificativa para esta pesquisa, o objetivo geral é aplicar a simulação computacional para análise da capacidade de atendimento de um novo restaurante universitário (RU).

Para cumprir esse objetivo, o presente artigo é organizado da seguinte forma. Na seção 2 há o referencial teórico base para a condução da pesquisa. A seção 3 apresenta o método de pesquisa utilizado. Na seção 4 estão informações sobre os modelos conceitual e computacional desenvolvidos. A seção 5 expõe os resultados obtidos e, por fim, na seção 6, estão as considerações finais.

\section{Referencial teórico}

Nesta seção há a exposição dos principais referenciais teóricos considerados nesta pesquisa. Assim, são apresentados, nas próximas subseções, detalhes sobre simulação computacional, as etapas para execução de projetos de simulação e a técnica IDEF-SIM para elaboração de modelos conceituais.

\subsection{Simulação computacional}

A simulação computacional busca reproduzir o funcionamento de um sistema por meio da utilização de um modelo computacional para a avaliação do comportamento e melhoria de desempenho do sistema estudado. Dentre os motivos para se desenvolver estudos de simulação estão: é possível predizer resultados, é possível levar em conta as variâncias do sistema, a simulação gera uma solução total, e pode ser financeiramente viável, pois não são necessárias mudanças reais no sistema estudado antes de testada sua eficiência e eficácia [17].

Conforme apresentado, a simulação aborda a análise de sistemas e pode utilizar modelos computacionais. Um sistema é um conjunto de entidades que agem e interagem entre si visando o alcance de um objetivo em comum [18]. Os modelos computacionais buscam representar os sistemas e seu funcionamento. Um modelo computacional corresponde a um programa de computador no qual as variáveis representam de forma similar o comportamento estocástico e dinâmico do sistema real em análise [19]. O modelo deve ser desenvolvido para um fim específico e sua validação determinada de acordo com o propósito do modelo [20].

Revista Brasileira de Computação Aplicada (ISSN 2176-6649), Passo Fundo, v. 8, n. 2, p. 99-112, jul. 2016100 
Os sistemas a serem simulados podem obter diversas classificações, como apresentado na Figura 1 [21]. Tais classificações interferem na forma de condução do projeto de simulação [17].

Figura 1: Classificação dos sistemas para fins de modelagem e simulação

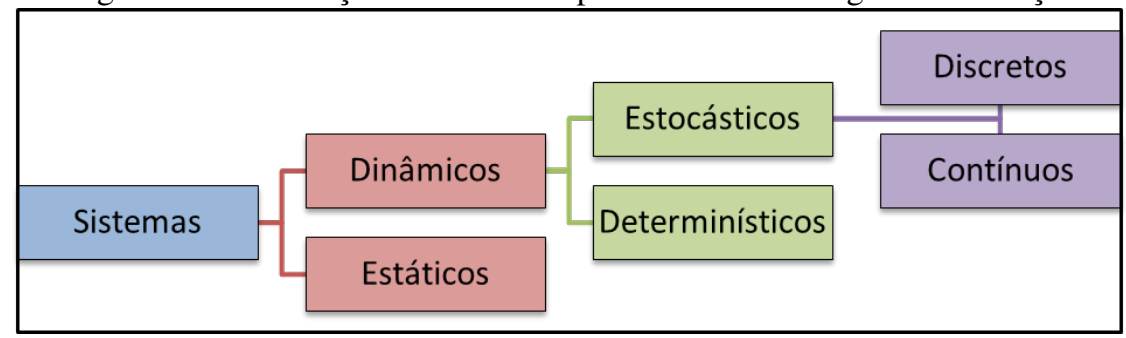

Fonte: adaptado de Harrell, Ghosh e Bowden [17].

A simulação estática não se baseia no tempo e geralmente envolve a geração de amostras aleatórias para gerar resultados estatísticos. Muitas vezes é chamada de simulação de Monte Carlo. De maneira oposta está a simulação dinâmica, que analisa as mudanças de estado do sistema à medida que ocorrem ao longo do tempo [17].

No contexto de sistemas dinâmicos, os estudos podem ser realizados de forma estocástica ou determinística. Na simulação determinística há entradas e saídas constantes, ou seja, sem aleatoriedade envolvida. Ao contrário, na simulação estocástica as entradas e saídas são aleatórias. Devido a isto, são necessárias diversas replicações para estimar o desempenho do sistema de forma precisa [17].

Quanto à diferenciação entre simulação de sistemas contínuos e sistemas discretos. Nesse último, as alterações de estado ocorrem somente em pontos bem definidos (pontos discretos), mantendo-se inalteradas ao longo de intervalos de tempo. Portanto, as mudanças de estado não ocorrem de modo contínuo [21].

Chwif e Medina [22] salientam que muitas vezes a técnica de simulação é interpretada de forma errônea e apontam que não pode ser confundida com: i) modelo matemático: pode-se usar fórmulas matemáticas no modelo, no entanto, não há como obter resultados a partir de uma forma analítica direta; ii) ferramenta estritamente de otimização: é uma ferramenta de análise de cenários e pode ser combinada com algoritmos de otimização; iii) bola de cristal: não pode prever o futuro; iv) substituta do pensamento inteligente: o ser humano é necessário no processo de tomada de decisão; v) panaceia: não é uma técnica que solucionará todos os problemas; vi) técnica de último recurso: não deve ser usada somente quando outras técnicas já falharam.

\subsection{Etapas para um projeto de simulação}

Vários autores sugerem passos para conduzir um estudo de simulação, dentre eles estão: Harrell, Ghosh e Bowden [17], Law [18], Shannon [19], Chwif e Medina [22], Emshoff e Sisson [23], Ingels [24], Pedgen, Shannon e Sadowski [25], Scriber [26] e Banks, Carson e Nelson [27]. No presente artigo convencionou-se o uso dos passos propostos por Harrell, Ghosh e Bowden [17] devido à similaridade entre os passos sugeridos por todos esses autores citados.

Harrell, Ghosh e Bowden [17] advogam que o processo de fazer simulação é interativo. As etapas devem ser seguidas, no entanto, algumas delas podem ser conduzidas em paralelo caso haja necessidade. As etapas propostas por esses autores encontram-se na Figura 2 e sua explicação é dada a seguir.

A etapa 1 consiste na definição dos objetivos, escopo e requisitos. São definidos o propósito/objetivo do estudo de simulação e o escopo do projeto. O planejamento do projeto deve levar em consideração os recursos, o tempo e o orçamento necessários para a execução do projeto. Quanto à etapa 2, ela refere-se à coleta e análise dos dados do sistema. É necessário identificar, coletar e analisar os dados que definem o sistema a ser modelado. Como resultados dessa etapa têm-se o modelo conceitual do sistema e os dados documentados.

$\mathrm{Na}$ etapa 3, há a construção do modelo computacional referente ao sistema em estudo. Já na etapa $4 \mathrm{o}$ modelo é validado, é quando há a verificação de falhas em sua elaboração para garantir uma representação fiel ao sistema. A condução dos experimentos é o foco da etapa 5, em que o modelo é executado para cada um dos cenários 
Figura 2: Etapas para o processo de simulação e o processo iterativo

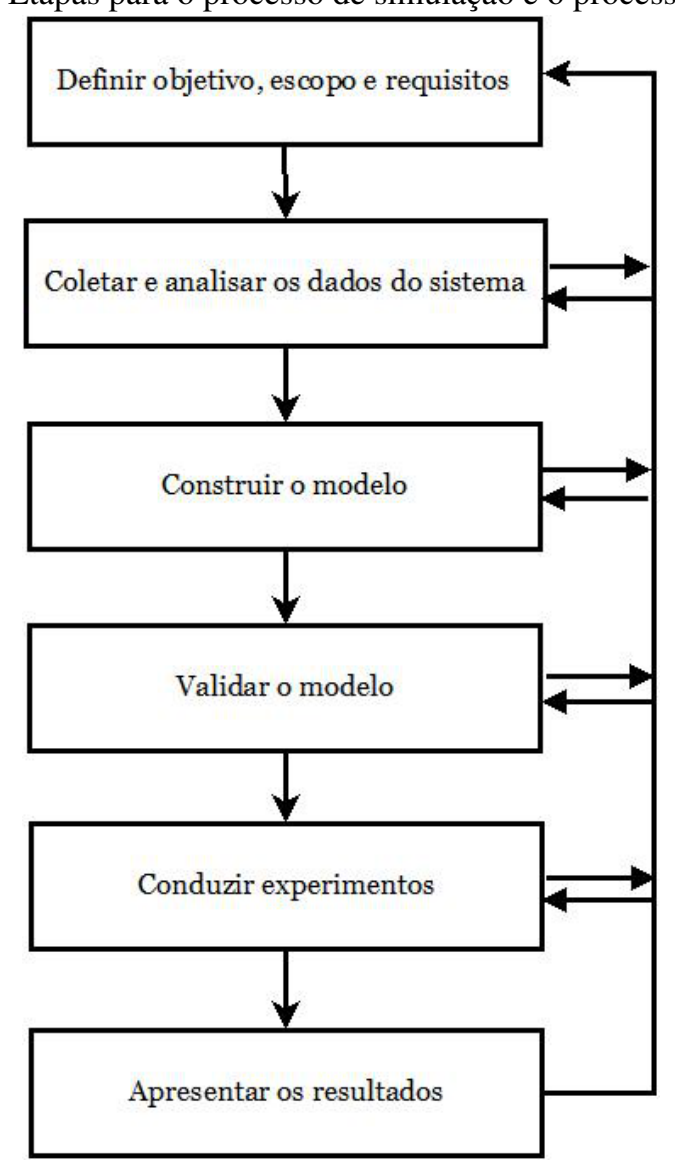

Fonte: adaptado de Harrell, Ghosh e Bowden [17].

estudados para avaliação e análise dos resultados. Na etapa 6 , há a apresentação dos resultados encontrados e as recomendações são feitas para que sirvam como base para o processo decisório.

\subsection{Modelagem conceitual e o método IDEF-SIM}

Os modelos conceituais são representações particulares de um sistema, podendo ser representações lógicas, matemáticas ou verbais (imitadores) [20]. Ressalta-se que o sistema a ser estudado pode ainda não estar em funcionamento e que a modelagem conceitual deve representar o sistema de maneira simplificada e com objetivos claros [28].

O modelo conceitual não é específico de um software e trata da base para a formulação do modelo computacional. O modelo computacional é a representação do modelo conceitual em uma linguagem de programação qualquer, e o modelo conceitual apresenta a função de documentar a base para o modelo computacional, daí sua importância [28].

Há diversas técnicas utilizadas na modelagem conceitual, porém poucas fornecem a base necessária ao projeto de simulação. Partindo dessa lacuna e da importância da modelagem conceitual para o êxito dos projetos de simulação computacional, Leal, Almeida e Montevechi [29] e Montevechi et al. [30] apresentam uma nova técnica para modelagem conceitual, nomeada de IDEF-SIM.

IDEF-SIM é uma técnica de modelagem conceitual que tem como base a fusão das técnicas IDEF0 (Integration Definition for Function Modeling) e IDEF3 com elementos de fluxograma. A técnica foi denominada IDEF-SIM e tem como foco o projeto de simulação, porém, é compatível com outros fins, tais como projetos de

Revista Brasileira de Computação Aplicada (ISSN 2176-6649), Passo Fundo, v. 8, n. 2, p. 99-112, jul. 2016102 
melhoria em geral [29]. A simbologia utilizada na técnica IDEF-SIM é apresentada na Figura 3 [29] [30].

Figura 3: Simbologia utilizada pela técnica IDEF-SIM

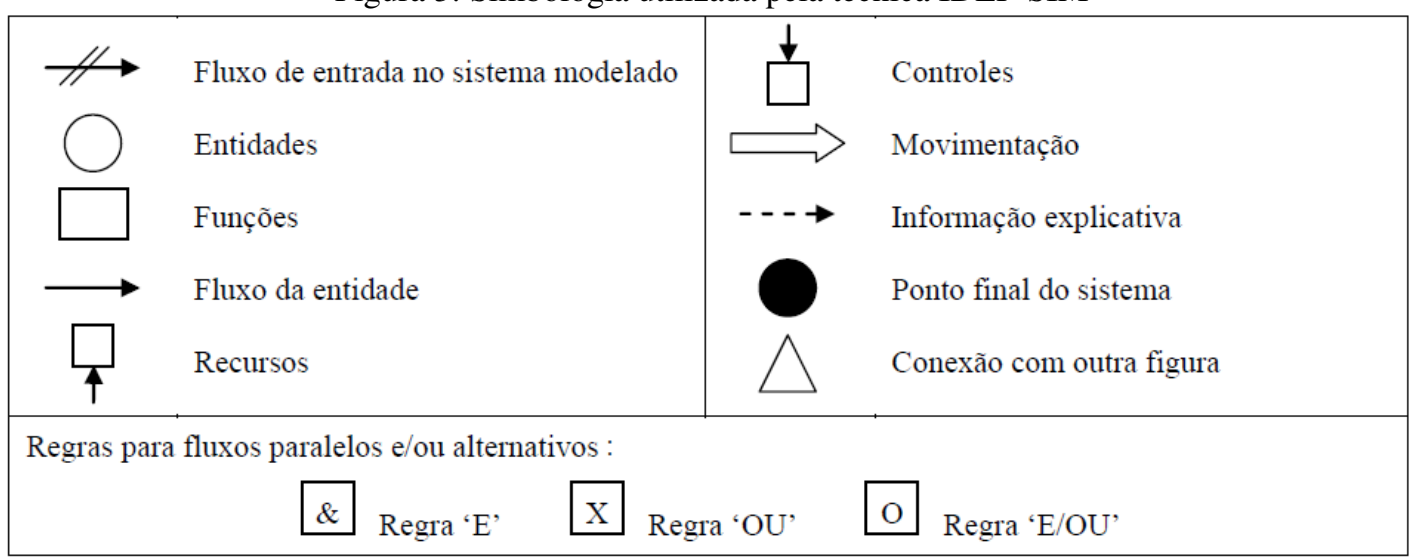

Fonte: adaptado de Leal, Almeida e Montevechi [29] e Montevechi et al. [30].

A função de cada elemento apresentado na Figura 3 é descrita a seguir [29]:

- Conexão com outra figura: utilizado em casos de divisão do modelo em figuras diferentes;

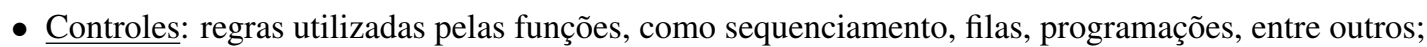

- Entidades: itens que sofrerão o processamento no sistema. Podem ser agrupadas ou divididas durante o processamento, como movimentação dada por meios próprios ou por meio da utilização de recursos. Quando representado, o símbolo somente será novamente utilizado na criação de uma nova entidade;

- Fluxo da entidade: caracterizam as entradas e saídas das entidades nas funções. Dão o direcionamento da entidade dentro do modelo;

- Fluxo de entrada e saída modelado: define a entrada ou criação de entidades no modelo;

- Funções: representam os locais de processamento ou movimentação das entidades. As funções podem modificar uma entidade ou alterar o tempo da entidade no fluxo;

- Informação explicativa: utilizada para inserir explicações no modelo visando facilitar o entendimento do mesmo;

- Movimentação: representa o deslocamento da entidade, se tal deslocamento possui importância sobre o modelo estudado, e deve apresentar os recursos e/ou tempo necessários para a movimentação;

- Ponto final do sistema: utilizado para a definição do ponto final de um caminho dentro do fluxo modelado;

- Recursos: representam os elementos utilizados para movimentação das entidades e execução de funções. Podem representar pessoas ou equipamentos, e podem ser estáticos ou dinâmicos;

- Regras de fluxo paralelo e/ou alternativos: após a execução de uma função dois caminhos podem ser executados paralelamente (regra E), ou de maneira alternativa (regra OU), ou permitindo ambas as regras (regra $\mathrm{E} / \mathrm{OU})$.

\section{Método de pesquisa}

A abordagem utilizada nesta pesquisa foi quantitativa. Essa abordagem considera que tudo pode ser quantificável, fazendo uma tradução em números de opiniões e informações para que sejam classificadas e analisadas estatisticamente [31]. 
Quanto ao procedimento de pesquisa, foi empregado o experimental. A finalidade deste procedimento é verificar as relações de causa e efeito, de forma que o pesquisador possa manipular as variáveis independentes, averiguando as alterações ocorridas nos resultados decorrentes dessas manipulações [32]. Esse procedimento é mais indicado para abordagens quantitativas. A pesquisa experimental é geralmente relacionada com experimentos controlados em laboratório e também com modelagens matemáticas e simulações computacionais [33]. Este procedimento foi utilizado nesta pesquisa devido ao uso de simulação.

A simulação computacional foi aplicada para representar o funcionamento do sistema estudado e para a verificação dos diversos cenários. O método faz uso de técnicas matemáticas e computacionais para descrever o funcionamento de um sistema produtivo com um todo ou parte do todo [34].

Para o processo de simulação foram seguidas as etapas propostas por Harrell, Ghosh e Bowden [17] e para a elaboração do modelo conceitual do sistema estudado foi utilizada a técnica IDEF-SIM [29] [30]. O IDEF-SIM foi utilizado por ser uma técnica que possui foco na simulação. Essa característica é desejável para a presente pesquisa, uma vez que permite a elaboração de modelos conceituais com as informações direcionadas e úteis para o desenvolvimento de modelos computacionais e para a documentação do modelo para entendimento do projeto [35]. Para a elaboração do modelo conceitual, execução dos experimentos e análise dos resultados, foi usado o software de simulação de eventos discretos ProModel ${ }^{\circledR}$ 2011, por este recurso estar disponível no departamento e pela maior afinidade dos pesquisadores.

O sistema analisado é o projeto de um restaurante universitário destinado ao uso pelos alunos de graduação de uma regional de uma universidade federal. O sistema é classificado como dinâmico, aleatório e discreto [17]. O cenário foi elaborado para analisar a capacidade de atendimento do sistema devido à grande afluência dos alunos, considerando que todos saíssem das aulas em horários próximos ao do almoço e se dirigissem ao RU. Também foi considerado o uso do RU pelos alunos de cursos de graduação que não frequentam aulas no período matutino.

O processo de coleta de dados foi dividido em três etapas e durou três meses. A primeira etapa foi iniciada com envio de e-mails e memorandos aos coordenadores de cursos de graduação. Após, a coleta de dados foi feita, presencialmente, com técnicos administrativos e coordenadores de cursos. Foram coletados os dados quanto ao número de alunos matriculados nos cursos de graduação, horário de saída das últimas turmas do período matutino e quantos alunos estão matriculados em cada uma das turmas do último horário do período matutino.

Na segunda etapa, foram coletados dados com o engenheiro responsável pela infraestrutura da regional da universidade. Foram obtidas informações sobre o projeto do RU, como a capacidade projetada e a planta baixa. A terceira etapa da coleta de dados foi realizada em um restaurante popular da cidade. Devido ao sistema em análise não estar em funcionamento, houve a necessidade de se coletar dados do funcionamento de um sistema similar ao estudado. Os dados coletados foram referentes aos tempos de processamento das entidades em cada um dos pontos de confluência importantes.

Esses dados coletados foram utilizados para a definição de distribuições probabilísticas referentes ao tempo de processamento das entidades nos seguintes locais: caixa, self service, distribuição e refeitório. O tempo de processamento desses locais foram representados pela distribuição triangular. Essa distribuição foi selecionada, pois não se conhecia a forma exata da distribuição para o sistema real, embora, as estimativas para o menor valor, maior valor e o valor mais provável de ocorrência fossem conhecidos [22]. Também foram coletados dados referentes ao tempo de passagem nas roletas de entrada e do refeitório. No modelo desenvolvido, esses tempos foram considerados constantes devido ao baixíssimo coeficiente de variação dos tempos coletados.

O sistema foi modelado e analisado conforme sistemas terminais [21]. O cenário foi elaborado considerando o período de almoço (duração de três horas). Para análise dos dados, foi utilizado um intervalo de confiança de 95\%. O valor do semi-intervalo de confiança, ou half-width, é interpretado com a confiança de que $95 \%$ das replicações obtêm uma média que estará no intervalo da média obtida mais ou menos o valor do semi-intervalo [36]. Foram realizadas trinta replicações. Os half-widths encontrados foram menores que $10 \%$ dos valores das médias amostrais, mostrando que a quantidade de replicações realizadas é satisfatória para a precisão dos dados.

Revista Brasileira de Computação Aplicada (ISSN 2176-6649), Passo Fundo, v. 8, n. 2, p. 99-112, jul. 2016104 


\section{Modelos conceitual e computacional}

A partir do modelo conceitual desenvolvido com base na técnica IDEF-SIM foi possível determinar, por exemplo, os componentes e as interações lógicas que compõem o sistema. O modelo conceitual é apresentado na Figura 4.

Figura 4: Modelo conceitual desenvolvido

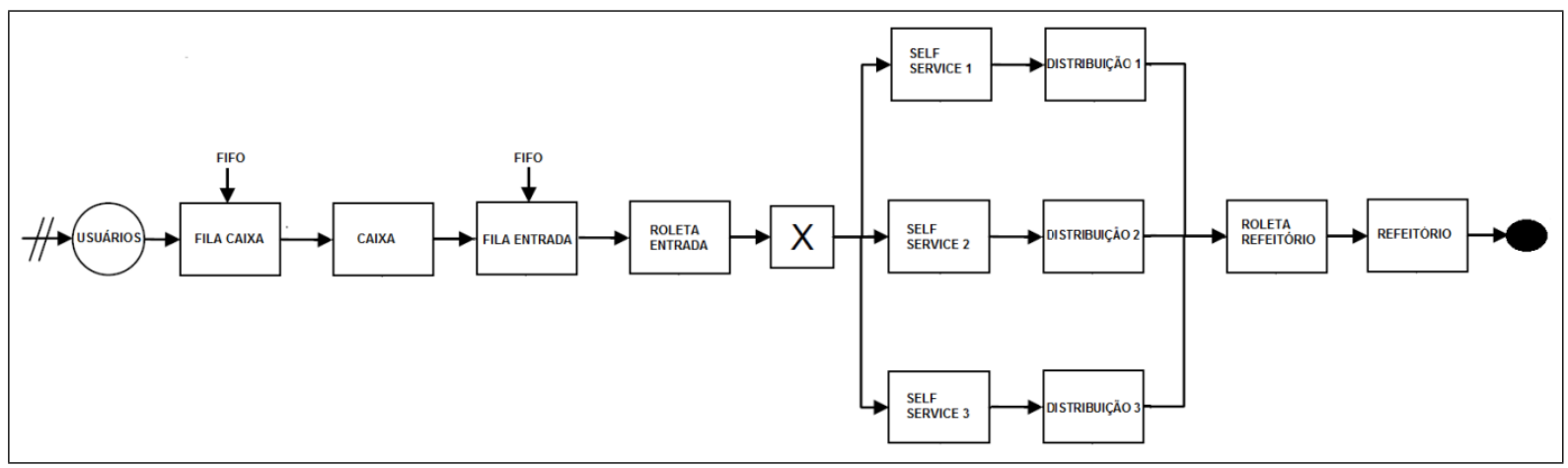

Fonte: elaborado pelos autores com dados da pesquisa.

De acordo com o modelo conceitual desenvolvido, a entidade USUÁRIOS ingressará no sistema primeiramente no local FILA CAIXA. Ao passar pela fila, a entidade sofrerá processamento no local CAIXA (pagamento do ticket) e se direcionará a outro local denominado FILA ENTRADA. Saindo da fila a entidade irá se dirigir ao local ROLETA ENTRADA. Ao passar pela roleta, a entidade poderá se direcionar ou ao SELF SERVICE 1 ou ao SELF SERVICE 2 ou ao SELF SERVICE 3, para se servir com a quantidade desejada dos alimentos disponíveis no buffet. Ao fim do processamento em um dos locais SELF SERVICE, a entidade irá se dirigir ao local DISTRIBUIÇÃO correspondente para que sejam servidos os alimentos com quantidades controladas (ex. carnes). Ao final do processamento nos locais de distribuição, as entidades dirigem-se ao local ROLETA REFEITÓRIO e em seguida ao REFEITÓRIO, onde farão as refeições e logo após sairão do sistema. A disciplina da fila dos locais FILA CAIXA e FILA ENTRADA é o FIFO (first in, first out - primeiro a entrar, primeiro a sair), ou seja, a primeira entidade que chegar ao local será processada primeiro.

O modelo conceitual elaborado foi validado pelos especialistas que possuem conhecimento sobre o funcionamento do restaurante universitário foco deste estudo. A validação foi realizada face a face (presencial) por meio da análise do modelo gráfico, como proposto por [20], e com o objetivo de fazer a aceitação ou não do modelo como representação do sistema projetado.

No início da elaboração do modelo computacional, a planta baixa do RU foi adaptada, deixando-se somente as partes necessárias para o estudo. Esse procedimento tornou o modelo mais enxuto e facilitou a visualização do processamento.

O próximo passo foi a definição dos locais no software de simulação. Para o modelo foram criados os seguintes locais: REFEITORIO, CAIXA, FILA_CAIXA, FILA_ENTRADA, ROLETA_ENTRADA, SELF_SERVICE_1, SELF_SERVICE_2,SELF_SERVICE_3, ROLETA_REFEITORIO, DISTRIBUIÇAO_1, DISTRIBUIÇAO_2 e DISTRIBUIÇAO_3. A Figura 5 apresenta parte dos registros dos locais feitos no software de simulação. Nota-se que foi necessário definir o nome dos locais, sua capacidade de processamento (Cap), a quantidade de cada local (Unidade), qual técnica estatística é utilizada para análise dos dados (o padrão do software é série de tempo) e as regras de decisão para acesso ao local. Por exemplo, o local REFEITORIO tem a capacidade de atender 304 usuários, havendo somente um refeitório. A técnica estatística utilizada na análise dos dados coletados foi série de tempo e a regra de decisão foi o mais velho.

A etapa seguinte foi a definição da entidade que sofrerá o processamento nos locais. Para o modelo computacional criado foi definida a entidade USUARIOS, que tem a velocidade de 50 metros por minuto (para desloca-

Revista Brasileira de Computação Aplicada (ISSN 2176-6649), Passo Fundo, v. 8, n. 2, p. 99-112, jul. 2016105 
Figura 5: Exemplo do registro dos locais feito no software de simulação

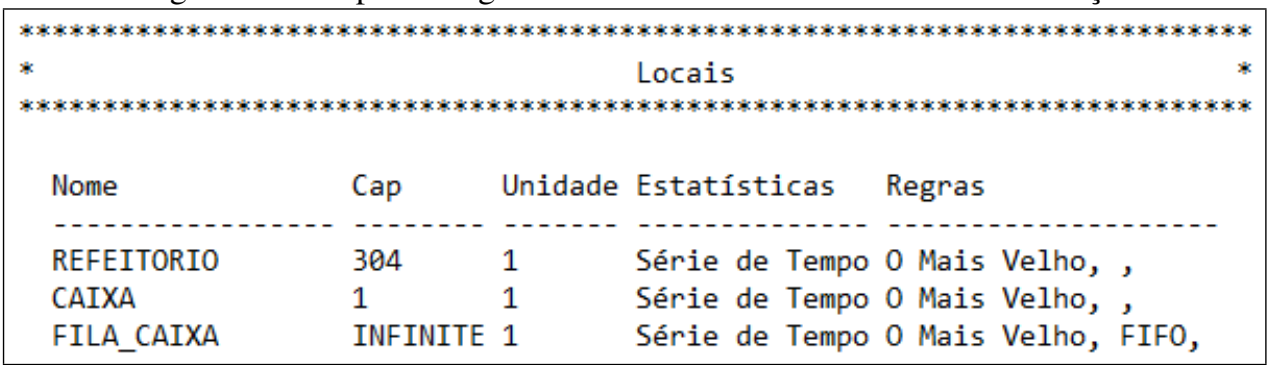

Fonte: elaborado pelos autores com dados da pesquisa.

mento calmo em ambiente fechado e com necessidade de atenção) e a técnica estatística utilizada para análise dos dados coletados nas replicações foi série de tempo (vide Figura 6).

Figura 6: Exemplo do registro da entidade

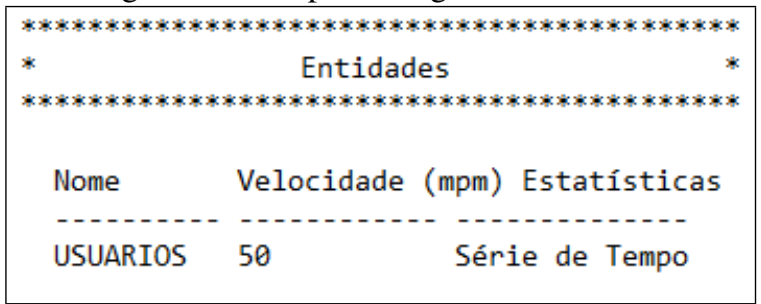

Fonte: elaborado pelos autores com dados da pesquisa.

Depois de registrada a entidade, as chegadas foram cadastradas conforme as informações do modelo conceitual e com base nos dados coletados com as coordenações dos cursos de graduação. As entidades foram alocadas no primeiro local do fluxo de processos (FILA_CAIXA), como pode ser observado no modelo conceitual apresentado. Salienta-se novamente que o intuito do modelo era verificar a capacidade de atendimento do RU em um cenário de utilização em massa pelos alunos de graduação.

Com isso, foram definidas as entidades que chegarão ao local, qual o local de chegada, a quantidade de entidades, quando ocorrerá a primeira chegada, a quantidade de ocorrências da chegada e a lógica utilizada na chegada. Como pode ser visto na Figura 7, os 54 alunos de uma turma de um dos cursos da universidade chegaram na FILA_CAIXA no tempo zero da simulação (logo no início do funcionamento do RU). Essa chegada ocorrerá somente uma vez, pois foi simulado um dia de funcionamento do RU. Portanto, não houve a necessidade de se informar a frequência, uma vez que todos os alunos dessa turma se direcionaram diretamente para o RU logo após serem liberados de suas aulas do período da manhã. No campo 'lógica' foi especificada a imagem que era utilizada para representar esses alunos de determinado curso.

Figura 7: Exemplo do registro das chegadas

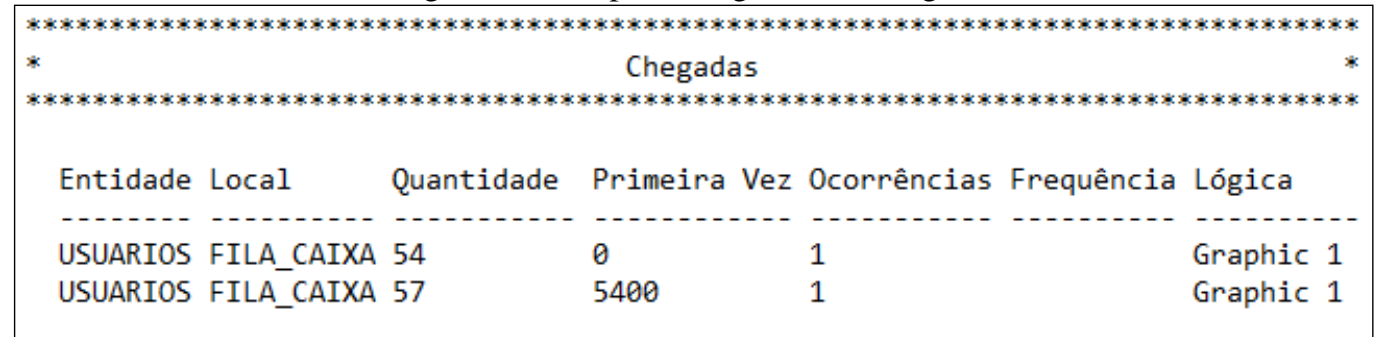

Fonte: elaborado pelos autores com dados da pesquisa.

Revista Brasileira de Computação Aplicada (ISSN 2176-6649), Passo Fundo, v. 8, n. 2, p. 99-112, jul. 2016106 
Também foram registrados os processamentos, por meio das lógicas de processo e de roteamento. Nesta fase de elaboração do modelo computacional os tempos de processamento em cada um dos locais são atribuídos de acordo com as distribuições de probabilidade geradas na coleta de dados, os tempos de movimentação entre os locais, o fluxo de processamento da entidade nos locais e outras regras de processamento.

Como pode ser visto na Figura 8, como exemplo, os usuários ao chegarem na FILA_CAIXA são direcionados imediatamente para o local CAIXA. A regra FIRST 1 indica que a primeira entidade a chegar ao local será processada primeiro e que cada entidade processada continua sendo uma entidade após o processamento. Assim que os usuários são direcionados para o CAIXA, há uma operação com duração Triangular $(3,42,65)$ segundos. Após o pagamento no caixa, o usuário atendido é direcionado para a FILA_ENTRADA e há uma movimentação considerável de 20 segundos.

Figura 8: Exemplo do registro dos processos

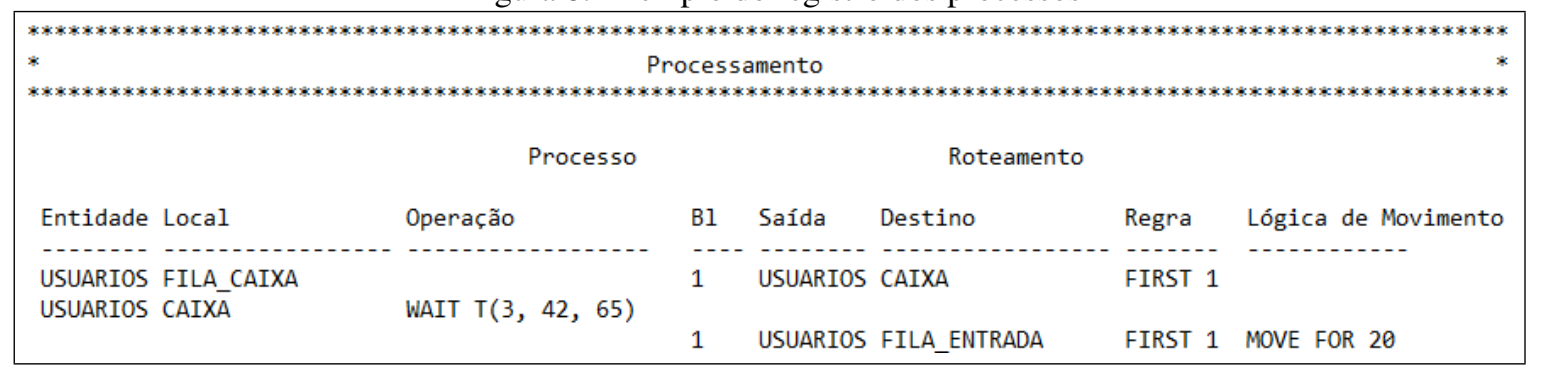

Fonte: elaborado pelos autores com dados da pesquisa.

A Figura 9 mostra o modelo em execução. Pode-se notar a chegada dos usuários na fila do caixa, em atendimento no caixa, movimentando-se na entrada de usuários, usuários já no buffet de distribuição dos alimentos e outros já realizando a refeição no refeitório.

Para a validação do modelo computacional foram seguidas as considerações de Sargent [20] e de Harrell, Ghosh e Bowden [17]. Considerando que o modelo representa um sistema em projeto, ou seja, não há a possibilidade de comparação dos resultados do modelo com os dados do sistema real (o que é esperado para aplicações de simulação em projetos), a validação prosseguiu da seguinte forma: o primeiro passo foi a comparação entre os modelos conceitual e computacional, e o segundo passo foi o rastreamento das entidades por meio do modelo. A primeira etapa é importante, pois o modelo computacional deve representar o mesmo fluxo de processamento do modelo conceitual por se tratar de representações do mesmo sistema. Além disso, a segunda etapa permitiu verificar se a lógica estava correta e se a precisão necessária foi mantida.

\section{Resultados obtidos}

Com base no cenário elaborado de utilização em massa do RU por alunos de graduação, verificou-se que em média 238 usuários, aproximadamente, dos 3.330 alunos de graduação que entraram no RU por meio da fila do caixa, seriam completamente atendidos nas três horas de funcionamento para almoço (veja Tabela 1). Isso representa aproximadamente $7,15 \%$ de atendimento da demanda total do cenário desenvolvido.

Observou-se que o recurso restritivo do sistema é o caixa, limitando o acesso à distribuição de alimentos. Como pode ser observado na Tabela 1, o tempo médio de permanência dos usuários na fila do caixa é de 45,71 minutos, atingindo o máximo de 90,67 minutos. A partir do momento em que o usuário é atendido no caixa, o tempo médio de permanência no sistema é de 27,55 minutos, alcançando o máximo de 34,15 minutos.

A Tabela 2 apresenta as porcentagens de utilização de cada um dos locais onde há o processamento de uma entidade por vez (capacidade única). Observa-se que o local CAIXA apresentou a maior porcentagem média de utilização $(98,97 \%)$. Devido a sua alta utilização, é sugerido o redimensionamento da capacidade de atendimento desse local.

Revista Brasileira de Computação Aplicada (ISSN 2176-6649), Passo Fundo, v. 8, n. 2, p. 99-112, jul. 2016107 
Figura 9: Animação do modelo conceitual desenvolvido

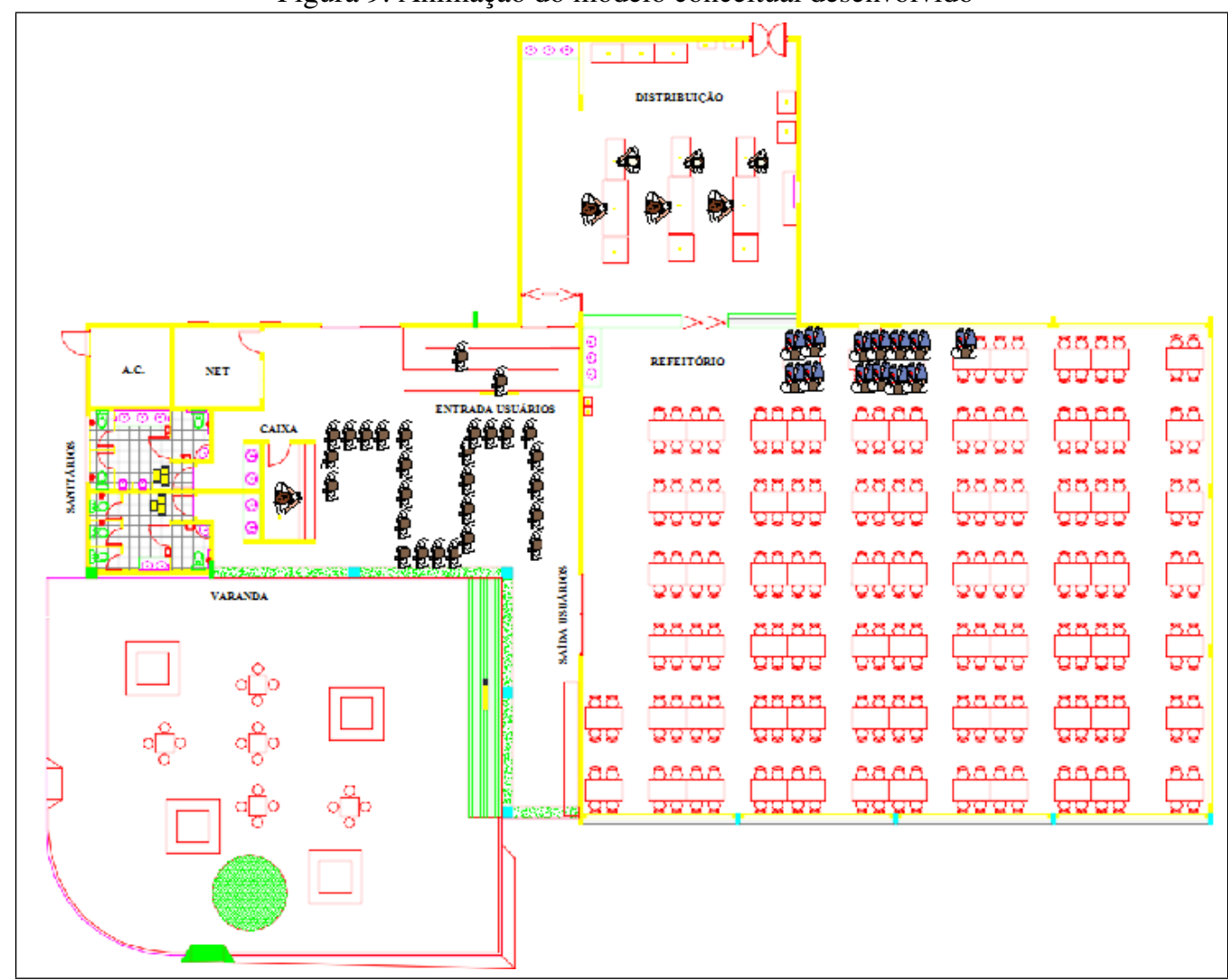

Fonte: elaborado pelos autores com dados da pesquisa.

Tabela 1: Algumas medidas de desempenho

\begin{tabular}{clcl}
\hline $\begin{array}{c}\text { Medida de } \\
\text { desempenho }\end{array}$ & $\begin{array}{l}\text { Total de saídas } \\
\text { (usuários) }\end{array}$ & $\begin{array}{c}\text { Tempo médio no sistema a } \\
\text { partir do caixa (minutos) }\end{array}$ & $\begin{array}{l}\text { Tempo médio na fila } \\
\text { do caixa (minutos) }\end{array}$ \\
\hline Média & 238,33 & 27,55 & 45,71 \\
Desvio-padrão & 6,03 & 0,53 & 0,53 \\
Half-Width & 2,25 & 0,20 & 0,20 \\
Mínimo & 226,00 & 0,00 & 0,00 \\
Máximo & 249,00 & 34,15 & 90,67 \\
\hline & Fonte: elaborado pelos autores com dados da pesquisa.
\end{tabular}

Tabela 2: Porcentagem de utilização dos locais de capacidade única

\begin{tabular}{clclcl}
\hline Local & Média & Desvio-padrão & Half-Width & Mínimo & Máximo \\
\hline CAIXA & 98,97 & 0,00 & 0,00 & 98,97 & 98,97 \\
SELF_SERVICE_1 & 79,84 & 1,45 & 0,54 & 76,76 & 83,29 \\
SELF_SERVICE_2 & 71,31 & 1,93 & 0,72 & 67,67 & 76,27 \\
SELF_SERVICE_3 & 51,79 & 4,33 & 1,62 & 42,50 & 58,48 \\
DISTRIBUICAO_1 & 3,98 & 0,18 & 0,07 & 3,62 & 4,34 \\
DISTRIBUICAO_2 & 3,51 & 0,19 & 0,07 & 3,13 & 3,90 \\
DISTRIBUICAO_3 & 2,56 & 0,23 & 0,09 & 2,18 & 2,96
\end{tabular}

Fonte: elaborado pelos autores com dados da pesquisa. 
Outros locais que tiveram maiores porcentagens médias de utilização foram o SELF_SERVICE_1 e o SELF_SERVICE_2, que apresentaram, em média, respectivamente, 79,84\% e 71,31\% de utilização. Os valores médios máximos de utilização atingidos nas replicações nesses locais foram de 83,29\% e 76,27\%, respectivamente. O local SELF_SERVICE_3 apresentou menor utilização média, comparado com os demais locais de self-service $(51,79 \%)$. Observa-se que esse local é necessário, mesmo com baixa utilização, pois ele reduzirá a utilização dos outros locais, evitando maior sobrecarga.

Quanto às porcentagens médias de utilização dos locais DISTRIBUICAO_1, DISTRIBUICAO_2 e DISTRIBUICAO_3, observa-se sua baixa utilização média dos mesmos: 3,98\%, 3,51\% e 2,56\%, respectivamente. No projeto elaborado, cada um desses locais está fisicamente ligado ao buffet self-service (vide Figura 9, área de distribuição). Neste caso, recomenda-se o redesenho do buffet.

Quanto ao refeitório, local onde os usuários farão as refeições, a capacidade total projetada foi para comportar 304 alunos. A Figura 10 mostra os estados desse local. Verificou-se que, em média, 96,84\% do tempo de funcionamento do RU, o local esteve ocupado por entidades e, em média, 3,16\% do tempo esteve completamente vazio. Em nenhum momento o local foi totalmente ocupado.

Figura 10: Estados do refeitório

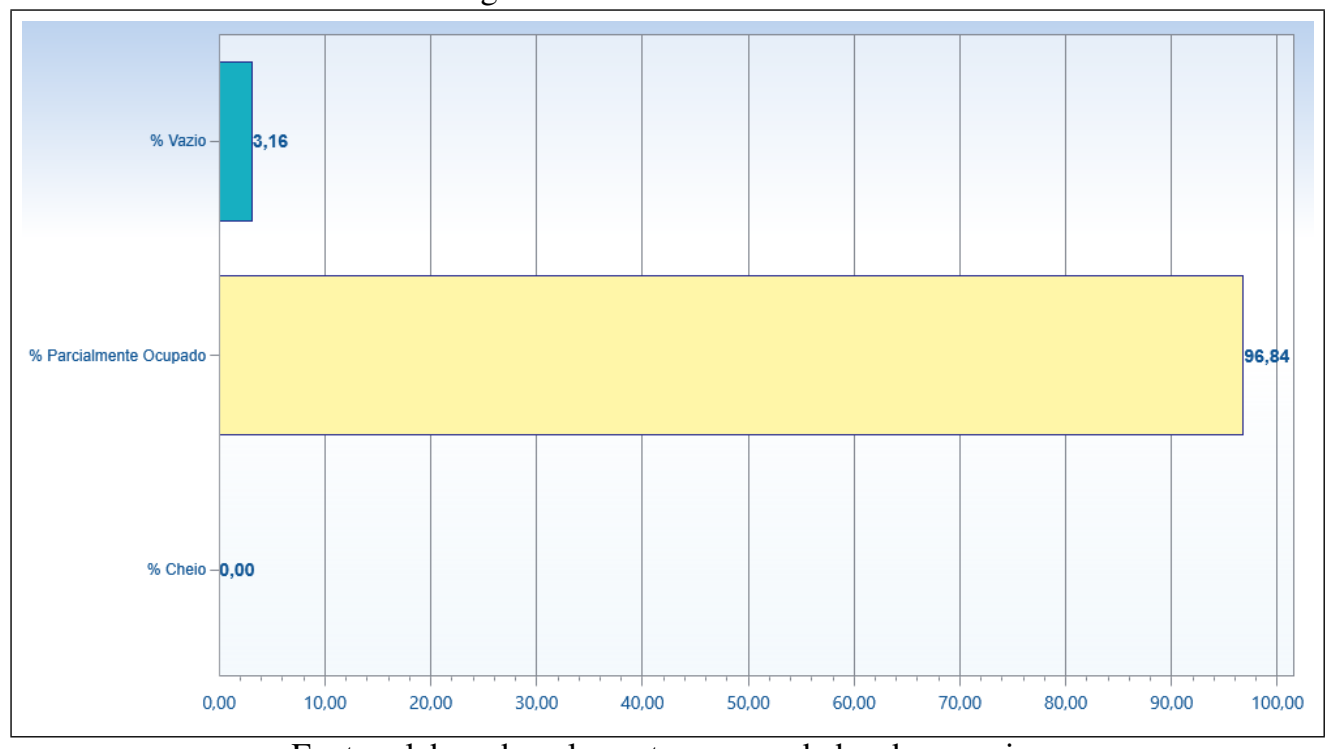

Fonte: elaborado pelos autores com dados da pesquisa.

Ainda quanto ao refeitório, a Tabela 3 expõe que 287,10 usuários, em média, acessaram o local durante o período de funcionamento. Esse valor atingiu o mínimo de 272 usuários e o máximo de 297. Comparando-se esse valor com o total de saídas (Tabela 1), nota-se que ainda haverá alunos realizando refeições no refeitório ao finalizar o horário de funcionamento do RU.

Tabela 3: Total de usuário que utilizaram o refeitório

\begin{tabular}{cccccc}
\hline Medida & Média & Desvio-padrão & Half-Width & Mínimo & Máximo \\
\hline $\begin{array}{c}\text { Média de usuários } \\
\text { no refeitório }\end{array}$ & 287,10 & 6,64 & 2,48 & 272 & 297 \\
\hline
\end{tabular}

Fonte: elaborado pelos autores com dados da pesquisa.

Revista Brasileira de Computação Aplicada (ISSN 2176-6649), Passo Fundo, v. 8, n. 2, p. 99-112, jul. 2016109 


\section{Considerações finais}

Neste trabalho, mediante o uso da técnica de simulação computacional, foi possível realizar a análise da capacidade de atendimento projetada para um novo restaurante universitário. Portanto, o objetivo almejado foi atingido. Para a análise, foi elaborado um cenário de utilização em massa do RU por alunos de graduação.

Verificou-se que o local CAIXA apresentou alta taxa de utilização, sendo esse um possível gargalo do sistema quando entrar em funcionamento. Em contrapartida, notou-se que os locais de distribuição controlada de alimentos possuíram baixa taxa de utilização. Também, identificou-se que, em média, o sistema conseguiu atender em torno de 7,15\% (238 alunos) da demanda do cenário elaborado (3.330 alunos), considerando o período de funcionamento de três horas do RU.

Sabe-se que, na prática, nem todos os alunos de graduação demandarão refeições no RU. No entanto, esse cenário demonstra que em uma situação de estresse do sistema (procura em massa pela população considerada), ele não seria capaz de atender todos os alunos durante o período de funcionamento (três horas para o horário de almoço).

Depois de analisados os resultados obtidos, é possível sugerir algumas melhorias no projeto do RU, a saber:

i) aumentar a capacidade do caixa, designando mais funcionários para o local quando constatada alta demanda;

ii) vender tickets alimentação em horários alternativos (fora do horário de almoço). Assim, a maioria dos alunos poderá seguir direto para a roleta de entrada do local de distribuição de alimentos, somente entregando o ticket para um funcionário antes de acessar a roleta;

iii) redesenhar o buffet para reduzir os locais de distribuição de alimentos controlados e, consequentemente, reduzir o número de funcionários alocados nesse espaço. Essa sugestão deve-se à identificação de baixa utilização do local.

Esta pesquisa contribui ao propiciar maior divulgação do tema simulação computacional e da possibilidade e importância do uso dessa técnica para análise de sistemas projetados. Ainda, auxilia na difusão da simulação computacional como uma técnica útil para o setor de serviços e não somente para a análise de sistemas de produção de bens.

Como sugestão de pesquisas futuras, tem-se: elaborar um estudo do redimensionamento do atendimento do caixa, para verificar a real necessidade de funcionários, considerando vários coeficientes de variação da demanda. Ainda, é possível realizar estudos futuros no RU visando intervenções que melhorem o seu funcionamento a partir de sua abertura, como análise do desempenho da cozinha e da gestão de estoques. Ressalta-se que há a possibilidade de realizar pesquisas futuras considerando outras aplicações de simulação computacional, como estudos para avaliação do impacto causado pela alteração de layout ou pela substituição de equipamentos em empresas, análise de desempenho de protocolos de roteamento em redes mesh, entre outros.

\section{Referências}

[1] GIRONIMO, G. D. et al. Virtual production planning of a high speed train using a discrete event simulation based approach. international journal on interactive design and manufacturing. International Journal on Interactive Design and Manufacturing (IJIDeM), Springer-Verlag, Bidart, v. 9, n. 1, p. 65-75, 2015. ISSN 1955-2513.

[2] ZAHRAEE, S. M.; CHEGENI, A.; ROHANI, J. M. Characterization of manufacturing system computer simulation using taguchi method. Jurnal Teknologi, Bogor, v. 72, n. 4, p. 77-82, 2015. ISSN 2180-3722.

[3] ROBINSON, S. et al. Facilitated modelling with discrete-event simulation: Reality or myth? European Journal of Operational Research, Amsterdam, v. 234, n. 1, p. 231-240, 2014.

[4] JOHANSSON, B.; KAISER, J. Turn lost production into profit - discrete event simulation applied on resetting performance in manufacturing systems. In: WINTER SIMULATION CONFERENCE. Proceedings... Los Alamitos: IEEE Computer Society, 2002. v. 2, p. 1.065-1.072. ISBN 0-7803-7614-5. 
[5] SHANNON, R. E. Introduction to the art and science of simulation. In: WINTER SIMULATION CONFERENCE, 30. Proceedings... Los Alamitos: IEEE Computer Society Press, 1998. p. 7-14. ISBN 0-78035134-7.

[6] JANSEN, D. R. et al. Simulation model of multi-compartment distribution in the catering supply chain. European Journal of Operational Research, Amsterdam, v. 133, n. 1, p. 210-224, 2001.

[7] TORGA, B. L. M. Modelagem, simulação e otimização em sistemas puxados de manufatura. Dissertação (Mestrado em Engenharia de Produção) — Universidade Federal de Itajubá, Itajubá, 2007.

[8] JAHANGIRIAN, M. et al. Simulation in manufacturing and business: a review. European Journal of Operational Research, Amsterdam, v. 203, n. 1, p. 1-13, 2010.

[9] SWART, W.; DONNO, L. Simulation modeling improves operations, planning and productivity of fast food restaurants. Interfaces, Hanover, v. 11, n. 6, p. 35-47, 1981.

[10] SÁNCHEZ, C. G.; RÍOS, R.; MALO, E. Enfoque híbrido simulación-proceso analítico jerárquico: Caso de estudio del rediseño de un restaurante. Revista de Métodos Cuantitativos para la Economía y la Empresa, Sevilla, v. 17, p. 23-41, 2014.

[11] FERREIRA, R. P. M. O método de monte carlo e a simulação de sistemas multiagentes. Abakós, Belo Horizonte, v. 1, n. 1, p. 89-99, 2012. ISSN 2316-9451.

[12] NUNES, S. E. Análise de impacto na transição entre protocolos de comunicação IPv4 e IPv6. Dissertação (Mestrado em Tecnologia) - Faculdade de Tecnologia da Universidade Estadual de Campinas, Campinas, 2013.

[13] PINOTTI, F. L. Simulação e emulação de tráfego multimedia em redes IP. Dissertação (Mestrado em Tecnologia e Inovação) — Faculdade de Tecnologia da Universidade Estadual de Campinas, Limeira, 2011.

[14] MENDES, A. et al. Combining heuristic procedures and simulation models for balancing a pc camera assembly line. Computers and Industrial Engineering, Paris, v. 49, n. 3, p. 413-431, 2005.

[15] RANKY, P. A simulation method and distributed server balancing results of networked industrial robots for automotive welding and assembly lines. Assembly Automation, Bingley, v. 23, n. 2, p. 192-197, 2003.

[16] VORST, J. G. van der; BEULENS, A. J.; BEEK, P. van. Modelling and simulating multi-echelon food systems. European Journal of Operational Research, Amsterdam, v. 122, n. 2, p. 354-366, 2000.

[17] HARRELL, C.; GHOSH, B. K.; BOWDEN, R. O. Simulation using ProModel. $3^{\text {rd }}$. ed. New York: McGraw Hill, 2012.

[18] LAW, A. M. Simulation modeling \& analysis. $4^{\text {th }}$. ed. New York: McGraw-Hill, 2007.

[19] SHANNON, R. E. System simulation: the art and Science. Englewood Cliffs: Prentice-Hall, 1975.

[20] SARGENT, R. G. Verification and validation of simulation models. In: WINTER SIMULATION CONFERENCE, 37. Proceedings... Orlando: Winter Simulation Conference, 2005. p. 130-143. ISBN 0-7803-95190 .

[21] FILHO, P. F. Introdução à modelagem e simulação de sistemas. 2. ed. Florianopólis: Visual Books, 2008.

[22] CHWIF, L.; MEDINA, A. C. Modelagem e simulação de eventos discretos: teoria e aplicações. 4. ed. Rio de Janeiro: Elsevier - Campus, 2015. ISBN 9788535279320.

[23] EMSHOFF, J. R.; SISSON, R. L. Design and use of computer simulation models. New York: Macmillan, 1970.

[24] INGELS, D. M. What every engineer should know about computer modeling and simulation. New York: Marcel Dekker, 1985.

Revista Brasileira de Computação Aplicada (ISSN 2176-6649), Passo Fundo, v. 8, n. 2, p. 99-112, jul. 2016111 
[25] PEDGEN, C. D.; SHANNON, R. E.; SADOWSKI, R. P. Introduction to simulation using siman. New York: McGraw Hill, 1990. 615 p.

[26] SCRIBER, T. J. An Introduction to Simulation Using GPSS/H. USA: John Wiley \& Sons, 1991. 437 p.

[27] BANKS, J.; CARSON, J. S.; NELSON, B. L. Discrete-event system simulation. New Jersey: Prentice Hall, 1996. $548 \mathrm{p}$.

[28] ROBINSON, S. Choosing the right model: Conceptual modelling for simulation. In: WINTER SIMULATION CONFERENCE. Proceedings... Phoenix: IEEE, 2011. p. 1.428-1.440. ISBN 978-1-4577-2108-3. ISSN 0891-7736.

[29] LEAL, F.; ALMEIDA, D.; MONTEVECHI, J. Uma proposta de técnica de modelagem conceitual para simulação através de elementos do idef. In: SIMPÓSIO BRASILEIRO DE PESQUISA OPERACIONAL. Anais... João Pessoa: SOBRAPO, 2008. p. 2.503-2.514.

[30] MONTEVECHI, J. A. B. et al. Conceptual modeling in simulation projects by mean adapted IDEF: an application in a brazilian tech company. In: WINTER SIMULATION CONFERENCE. Proceedings... Baltimore: IEEE, 2010. p. 1.624-1.635. ISBN 978-1-4244-9866-6. ISSN 0891-7736.

[31] SILVA, E. L.; MENEZES, E. M. Metodologia da pesquisa e elaboração de dissertação. 4. ed. Florianópolis: UFSC: [s.n.], 2005.

[32] CRESWELL, J. W. Research design: qualitative \& quantitative approaches. [S.1.]: Sage Publications, 1994. $228 \mathrm{p}$.

[33] BRYMAN, A. Research methods and organization studies. London: Routledge, 1989. 300 p. (Contemporary Social Research). ISBN 978-0415084048.

[34] NETO, R. M.; PUREZA, V. Modelagem e simulação, In: MIGUEL, P.A.C (Org.). Metodologia de pesquisa em engenharia de produção e gestão de operações. 2. ed. Rio de Janeiro: Campus-Elsevier, 2011.

[35] BATEMAN, R. E. et al. Simulação de sistemas: aprimorando processos de logística, serviços e manufatura. [S.1.]: Elsevier Brasil, 2013. ISBN 9788535271638.

[36] KELTON, W. D.; SADOWSKI, R. P.; SADOWSKI, D. A. Simulation with Arena. $4^{\text {th }}$. ed. London: McGrawHill, 2007. 